\title{
Lower limb muscle activities and gain in balancing ability following two types of stair gait intervention in adult post-chronic stroke patients: A preliminary, randomized-controlled study
}

\author{
Choi Yoon-Hee ${ }^{1}\left(\mathbb{D}\right.$, Kim Kyoung $^{2}\left(\mathbb{D}\right.$, Lee Sang-Yong ${ }^{3}\left(\mathbb{D}\right.$, Cha Yong-Jun ${ }^{4}$ (C) \\ ${ }^{1}$ Department of Physical Therapy, Graduate School, Daejeon University, Daejeon City, South Korea \\ ${ }^{2}$ Department of Physical, College of Rehabilitation Science, Daegu University, Daegu City, South Korea \\ ${ }^{3}$ Department of Physical Therapy, U1 University, Young-Dong, South Korea \\ ${ }^{4}$ Department of Physical Therapy, College of Health and Medical Science, Daejeon University, South Korea
}

Received: July 04, 2018 Accepted: October 23, 2018 Published online: March 03, 2020

\begin{abstract}
Objectives: This study aims to compare the changes in lower limb muscle activities after stair ascending and descending training at two different heights of stairs in patients with chronic stroke and to suggest a stair height which is more effective in improving the strength and balancing ability of these patients.

Patients and methods: Between November 2016 and February 2017, a total of 20 patients (14 males, 6 females; mean age 56 years; range, 52 to 61 years) with hemiparesis were included in this randomized-controlled study. The patients were randomly assigned to the $10-$ or $15-\mathrm{cm}$ stair height group (10- and $15-\mathrm{cm}$ groups, respectively; $\mathrm{n}=10$ in each). Both groups received comprehensive rehabilitation therapy and additionally performed stair gait training for $30 \mathrm{~min}$ four times per week for a total of six weeks. Balancing abilities and the activities of the paralyzed lower limb's rectus femoris, biceps femoris, tibialis anterior, and gastrocnemius during stair ascending were measured before and after the stair gait training.
\end{abstract}

Results: During stair ascent, the $15-\mathrm{cm}$ group showed significantly greater muscle activities of the rectus femoris, biceps femoris, and tibialis anterior than the $10-\mathrm{cm}$ group $\left(\mathrm{p}<0.001, \eta^{2}=0.115 ; \mathrm{p}=0.001, \eta^{2}=0.022\right.$; and $\mathrm{p}=0.001, \eta^{2}=0.036$, respectively).

Conclusion: Our study results suggest that ascending stairs with $15-\mathrm{cm}$ step height results in a greater muscle activity than with a $10-\mathrm{cm}$ step height.

Keywords: Balance, chronic stroke, muscle activity, stair gait training, stair height.

Stroke is a disease in which cerebral function is lost due to blockage of blood supply or bleeding of brain tissue. ${ }^{[1,2]}$ It is associated with various neurological dysfunctions that affect movement, sensation, postural control, language, cognition, and perception depending on the location of the brain lesion and degree of damage. ${ }^{[3,4]}$

The reduction in the walking ability of patients with hemiplegia is mainly caused by weakness of the lower limbs, ${ }^{[5]}$ which occurs in the flexor muscles of the ankle, knee, and hip joints; ${ }^{[6,7]}$ hence, strengthening of the leg muscles and improvement of function through effective management, such as strength and coordination, ${ }^{[8]}$ are the mainstays of the management.

Stairway walking is a functional activity which is frequently encountered in activities of daily living. ${ }^{[9]}$ Stairway walking is similar to walking on a flat ground in that there is a reciprocal movement of both legs and a double stance phase. ${ }^{[10]}$ However, since stairway ascending moves the center of gravity of the body in the anterior and vertical directions, more strength and postural control ability of the lower limb is needed than while walking on a level surface. ${ }^{[1]}$ A previous study has reported that knee joint extension moments

Corresponding author: Cha Yong-Jun, PT. Department of Physical Therapy, College of Health and Medical Science, Daejeon University, 34520 Daejeon, South Korea. e-mail: cha0874@gmail.com 
are up to two times greater during stair ascending than during level-ground walking. ${ }^{[12,13]}$ Therefore, stair walking training is a representative task-oriented training method for improving leg strength and postural control of patients with hemiparetic stroke in indoor and outdoor environments. ${ }^{[14]}$

Rehabilitation training for improving the stairclimbing function of patients with stroke is usually performed through the corner exercise staircase. ${ }^{[15]}$ However, some stairway walkers have different heights of 10 or $15 \mathrm{~cm},{ }^{[15,16]}$ and the regulation of the Welfare for the Disabled Law for stairway height of less than $18 \mathrm{~cm}$ is also vague, making it difficult to produce a consistent stairway walker. ${ }^{[17,18]}$ Therefore, the height of the stairway walker that would an opportunity to improve the function of the stroke patient should be proposed. Thus far, studies have been conducted on the effects of stair walking training to improve the function of patients with stroke. Comparative studies on the muscle activity of leg muscles during gait on the ground and on the stairs, ${ }^{[19]}$ lower limb muscle activity according to various foot contact areas during ascending the stairs ${ }^{[16]}$ and muscle activity of the lower extremity muscles while walking on ramps versus stairs have been conducted. ${ }^{[15,20]}$ However, no study has examined the balancing ability and muscle activity of the lower extremity muscles during stair ascending according to different stair heights.

In the present study, we hypothesized that stair walking training at stair heights of 10 and $15 \mathrm{~cm}$ would produce greater balance and muscle activity in the paretic lower limb, compared to the baseline variables and that there would be a difference in functional recovery between the two heights. Therefore, the aim of this study was to compare the lower limb muscles' activities and balancing ability using two different stairway heights for the walking training of patients with hemiparetic chronic stroke and to identify a stair height that is more effective in improving strength and balancing ability.

\section{PATIENTS AND METHODS}

This preliminary, randomized-controlled study was conducted in a tertiary hospital between November 2016 and February 2017. A total of 58 patients hospitalized with hemiparetic stroke were screened for the study. Inclusion criteria were as follows: having a diagnosis of hemiparesis for more than six months, being able to walk more than 10 meters independently on a flat ground without an assistive walking device such as a cane, being able to go up and down stairs independently with or without handrail support, being able to climb two stairs within five sec without using the handrails, and having a Mini Mental State Examination (MMSE)-Korean version score of $\geq 24$ points who could understand and follow the instructions given by the researcher. Those with other neurological or orthopedic conditions other than stroke and those whose paralyzed dorsiflexor had a muscle tone of Grade $\geq 2$ according to the modified Ashworth scale ${ }^{[21]}$ were excluded. A total of 35 patients did not meet the inclusion criteria and three patients refused to participate. Finally, 20 patients (14 males, 6 females; mean age 56 years; range, 52 to 61 years) with hemiparesis were included in the study. The study was conducted using a randomized design. For randomization, 20 sealed envelopes were prepared in advance and marked inside with $1(n=10)$ or $2(n=10)$, which indicated the $10-\mathrm{cm}$ group (stair walking training with stair height of $10 \mathrm{~cm}$ ) or the $15-\mathrm{cm}$ group (stair walking training with stair height of $15 \mathrm{~cm}$ ), respectively. An independent therapist not involved in the study performed the randomization.

A written informed consent was obtained from each patient. The study protocol was approved by Daejeon University Research Ethics Committee (1040647-201608-HR-002-03). The study was conducted in accordance with the principles of the Declaration of Helsinki.

\section{Interventions}

All patients underwent neurodevelopmental treatment consisting of joint mobilization, muscle stretching, and strengthening, and functional training for $30 \mathrm{~min}$ per day for five times per week. In addition, stairway walking training was performed for $30 \mathrm{~min}$, four times per week for a total of six weeks.

The stairway gait training was performed on a specially-made staircase with a width of $150 \mathrm{~cm}$, length of $28 \mathrm{~cm}$, and height of either 10 or $15 \mathrm{~cm}$. The stairway walk training was conducted with the following instruction: "Go up and down the stairs comfortably." When climbing the stairs, all patients were asked to walk in the order of affected foot followed by the lesser affected foot and to walk barefoot at a self-selected comfortable speed on a four-step wooden staircase. ${ }^{[16,22]}$ For safety purposes, the staircase was positioned with the patient's non-affected side next to a wall. The patients were instructed to walk in steps on the staircase without using the handrails. The therapist always supervised or minimally assisted the patient to prevent accidents such as falls. During stair climbing, the patients walked on level ground 
for 3 meters before stepping onto the staircase and continued walking to the end of an elevated walkway. When descending the stairs, the patients walked from the end of the elevated walkway and continued walking on level ground to the starting position. ${ }^{[4,22,23]}$

\section{Outcome measures}

Outcome measures were performed by an examiner who did not participate in the randomization and blinded to the patient groups. All tests were performed in a separate space in the physical therapy room.

The Berg balance scale (BBS) and Timed Up and Go (TUG) tests were performed to measure the patients' balancing ability. ${ }^{[24,25]}$

The MP150 surface electromyography (EMG) system (Biopac System Inc., CA, USA), which has a good reliability and validity, was used to measure the activities of the rectus femoris, biceps femoris, tibialis anterior, and gastrocnemius during stair ascending. ${ }^{[26,27]}$ To minimize skin resistance to the EMG signal of the muscle area, the hairs around the attachment area were removed. The skin stratum was, then, rubbed three or four times with a thin sandpaper, and finally, the skin was wiped with alcohol-soaked cotton. The Ag-Ag/Cl surface electrode mode (Biopac System Inc., CA, USA; diameter $2 \mathrm{~cm}$ ) was used for EMG signal acquisition. Each electrode was attached parallel to the muscle belly of the attachment muscle. ${ }^{[28]}$ The samples were secured at $1,000 \mathrm{~Hz}$ using a personal computer. The raw surface EMG signals were band-pass filtered between 30 and $500 \mathrm{~Hz}$ to minimize noise. The collected EMG signals were analyzed using the Acqknowledge 3.8.1 software (Biopac System Inc., CA, USA). For normalization of the EMG signal, the collected EMG signals were converted to root mean square (RMS), and for normalization of the RMS values, the percentage of maximum isometric contraction (\% MVIC) values, ranging from 0 to $100 \%$, were obtained by dividing the RMS values by the MVIC. For acquisition of MVIC data, the EMG electrodes were placed on the muscles and the patients were asked to perform four MVIC exercises against manual resistance provided by the researcher: (i) knee extension while sitting with hip and knee at $90^{\circ}$ of flexion, (ii) knee flexion in prone with knee at $90^{\circ}$ of flexion, (iii) ankle dorsiflexion while sitting with hip and knee at $90^{\circ}$ of flexion and ankle touching the floor, and (iv) ankle plantar flexion while standing on the affected leg. ${ }^{[29]}$ Joint angles in these positions were verified with a manual goniometer. The patients completed one or two warm-up isometric muscle contractions at 50 and $75 \%$ of their perceived maximal effort with one min of rest in between each muscle contraction. After each warm-up isometric muscle contraction, the patients completed five sec maximal

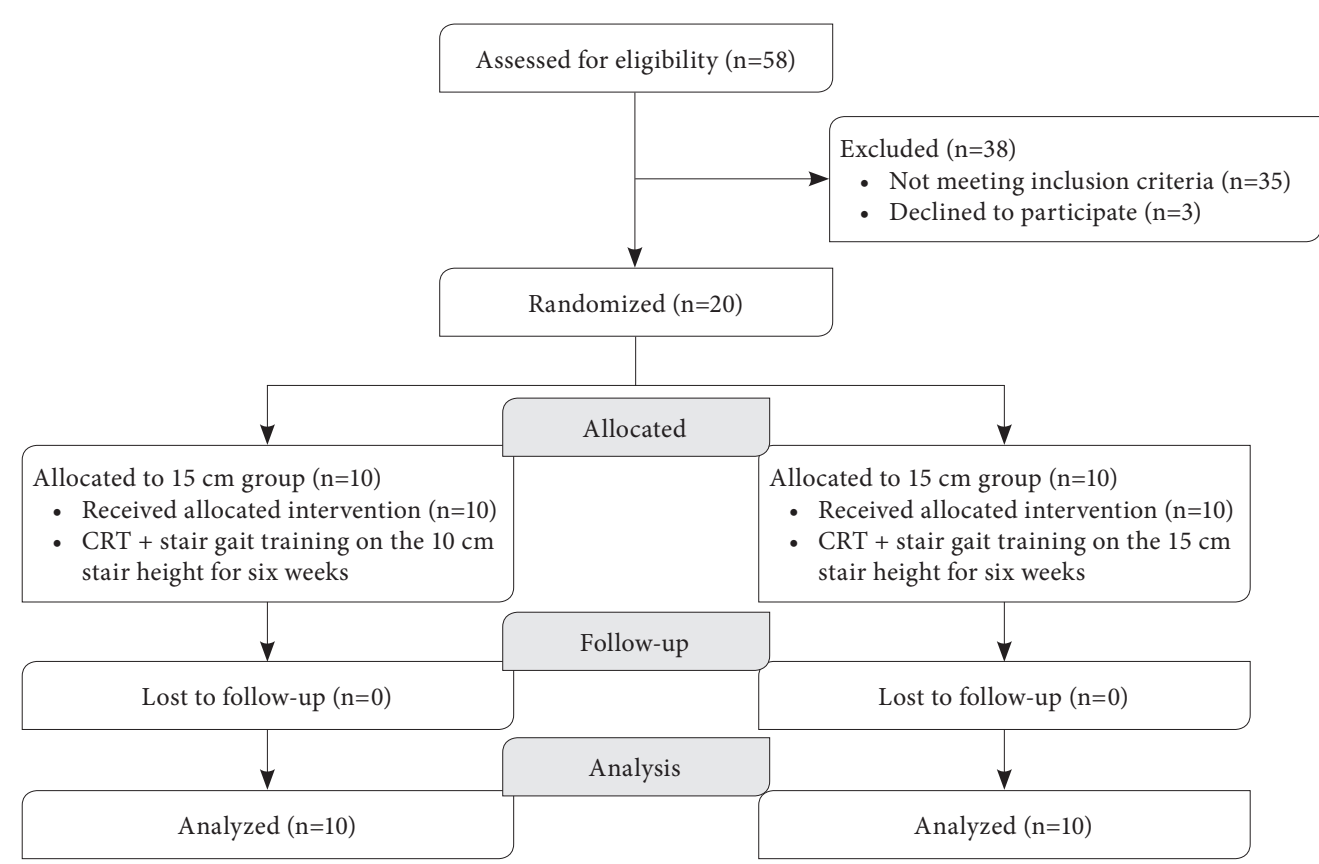

Figure 1. CONSORT diagram showing study flow.

CRT: Comprehensive rehabilitation therapy. 
isometric leg (e.g., knee extension, knee flexion, ankle dorsiflexion, and plantar flexion, respectively) three times with one min of rest between the attempts to avoid fatigue. ${ }^{[30]}$ The mean value was calculated, and EMG data were excluded for one sec before and one sec after the completion of each attempt.

In this study, the time required for the two-step climbing was approximately five sec. Considering this, we measured the muscle activity for stance and swing phase for seven sec during stair ascending on usually used stairs (width: $150 \mathrm{~cm}$; length: $25 \mathrm{~cm}$; height: $12 \mathrm{~cm}$ ), and the data for five sec, except the first and last seconds, were used for data analysis.

All variables were measured before and after stair walking training for six weeks. All data measured before and after six weeks of training were collected three times for each measurement variable, and the mean value was finally calculated. To minimize fatigue induced by several measurements, an adequate rest time was given between the measurements. If the patient complained of fatigue during the measurement or asked for more breaks, the measurement was discontinued and additional rest was given.

\section{Statistical analysis}

Statistical analysis was performed using the IBM SPSS for Windows version 23.0 software (IBM Corp., Armonk, NY, USA). Descriptive data were expressed in mean \pm standard deviation (SD) or number and frequency. The Shapiro-Wilk test was used to test the data for normality. To compare the general characteristics at baseline between the two groups, independent t-test was used to analyze continuous variables and the Fisher's exact test was used to analyze dichotomous variables. Paired t-tests were conducted for each significant time for the main effects to identify the source of the significant difference between pre- and post-training. Two-way repeated measures analysis of the variance (ANOVA) was carried out to compare the mean differences of BBS and TUG between the two groups (10- and $15-\mathrm{cm}$ groups) as the first factor and time (pre- and post-training) as the second factor. For variables with a significant difference at baseline test between the two groups, analysis of covariance (ANCOVA), i.e., controlling for the pretest difference, was used to examine which group showed a significant better post-test performance when there was a statistical significance in the pretest of each outcome variable

\begin{tabular}{|c|c|c|c|c|c|}
\hline \multicolumn{6}{|c|}{$\begin{array}{l}\text { TABLE } 1 \\
\text { Baseline demographic and clinical characteristics of patients }\end{array}$} \\
\hline & \multicolumn{2}{|c|}{ 10-cm group } & \multicolumn{2}{|c|}{ 15-cm group } & \multirow[b]{2}{*}{$p$} \\
\hline & $\mathrm{n}$ & Mean \pm SD & $\mathrm{n}$ & Mean \pm SD & \\
\hline Sex & & & & & 1.000 \\
\hline Male & 7 & & 7 & & \\
\hline Female & 3 & & 3 & & \\
\hline Age (years) & & $57.4 \pm 10.4$ & & $56.0 \pm 10.0$ & 0.752 \\
\hline Height $(\mathrm{cm})$ & & $162.9 \pm 7.7$ & & $164.5 \pm 9.2$ & 0.679 \\
\hline Weight (kg) & & $60.8 \pm 7.3$ & & $63.1 \pm 9.2$ & 0.545 \\
\hline Paretic side & & & & & 1.000 \\
\hline Right & 6 & & 6 & & \\
\hline Left & 4 & & 4 & & \\
\hline Onset duration (month) & & $30.5 \pm 9.9$ & & $33.3 \pm 9.9$ & 0.535 \\
\hline Stroke type (infarction/hemorrhage) & $6 / 4$ & & $7 / 3$ & & 1.000 \\
\hline Berg balance scale (score) & & $29.8 \pm 4.7$ & & $33.2 \pm 4.4$ & 0.114 \\
\hline Timed up and go (sec) & & $17.6 \pm 3.3$ & & $16.2 \pm 2.0$ & 0.281 \\
\hline \multicolumn{6}{|l|}{ Muscle activity } \\
\hline Rectus femoris & & $35.8 \pm 0.96$ & & $38.8 \pm 0.5$ & 0.012 \\
\hline Biceps femoris & & $26.2 \pm 1.2$ & & $32.0 \pm 1.0$ & 0.002 \\
\hline Tibialis anterior & & $16.4 \pm 0.96$ & & $27.3 \pm 1.0$ & $<0.001$ \\
\hline Gastrocnemius & & $38.5 \pm 0.83$ & & $49.9 \pm 0.9$ & $<0.001$ \\
\hline
\end{tabular}




\begin{tabular}{|c|c|c|c|c|c|c|c|}
\hline \multicolumn{8}{|c|}{$\begin{array}{c}\text { TABLE } 2 \\
\text { Comparison of functional evaluation results between the two groups }\end{array}$} \\
\hline & \multicolumn{2}{|c|}{$10-\mathrm{cm}$ group $(\mathrm{n}=10)$} & \multicolumn{2}{|c|}{$15-\mathrm{cm}$ group $(\mathrm{n}=10)$} & \multirow[b]{3}{*}{ F (time $\times$ group $)$} & \multirow[b]{3}{*}{ F (ANCOVA) } & \multirow[b]{3}{*}{$p$} \\
\hline & Preoperative & Postoperative & Preoperative & Postoperative & & & \\
\hline & Mean \pm SD & Mean \pm SD & Mean \pm SD & Mean \pm SD & & & \\
\hline Berg balance scale (score) & $29.8 \pm 4.7$ & $32.9 \pm 3.6 \dagger$ & $33.2 \pm 4.4$ & $36.0 \pm 4.0 \dagger$ & 0.134 & - & 0.007 \\
\hline Timed Up and Go (sec) & $17.6 \pm 3.3$ & $15.1 \pm 2.4 \dagger$ & $16.2 \pm 2.0$ & $14.8 \pm 1.9 \dagger$ & 2.151 & - & 0.107 \\
\hline \multicolumn{8}{|l|}{ Muscle activity } \\
\hline Rectus femoris & $35.8 \pm 1.0$ & $34.5 \pm 1.0$ & $38.8 \pm 0.5$ & $40.5 \pm 0.8 \dagger$ & - & $29.544^{*}$ & 0.115 \\
\hline Biceps femoris & $26.2 \pm 1.2$ & $27.8 \pm 1.0$ & $32.0 \pm 1.0$ & $34.9 \pm 0.9 \dagger$ & - & $16.481^{*}$ & 0.022 \\
\hline Tibialis anterior & $16.4 \pm 1.0$ & $17.1 \pm 1.1$ & $27.3 \pm 1.0$ & $29.6 \pm 0.8$ & - & $16.716^{*}$ & 0.036 \\
\hline Gastrocnemius & $38.5 \pm 0.8$ & $44.0 \pm 0.8 \dagger$ & $49.9 \pm 0.9$ & $51.5 \pm 0.9$ & - & 3.286 & 0.255 \\
\hline
\end{tabular}

SD: Standard deviation; Muscle activity (\% of maximal voluntary isometric contraction); ${ }^{\star}$ Significantly different as controlling for the pretest difference ( $\left.<<0.05\right)$; $\dagger$ Significantly different compared to baseline $(\mathrm{p}<0.05)$.

between the two groups. A $p$ value of $<0.05$ was considered statistically significant. An eta-squared $\left(\eta^{2}\right)$ was calculated to identify the magnitude of the effect sizes, and it was interpreted as small (0.02), medium (0.13), or large $(0.26) .^{[31,32]}$

\section{RESULTS}

The study flow chart is shown in Figure 1. All patients, 10 in the $10-\mathrm{cm}$ group and 10 in the $15-\mathrm{cm}$ group, completed the six-week intervention. No significant differences in the pretest demographic and clinical characteristics were found between the two groups, except for the muscle activities (Table 1).

Table 2 provides a summary of the balance and muscle activity parameters before and after training in the two groups. A significant improvement in the BBS and TUG was observed after stair walking training in the two groups $(p<0.05)$; however, there were no significant difference in the time $\times$ group interaction. The ANCOVA for the muscle activities showed significant and large effects. After the intervention, the $15-\mathrm{cm}$ group had significantly greater rectus femoris, biceps femoris, and tibialis anterior activities ( $F[1,18]=29.544, \mathrm{p}<0.001, \eta^{2}=0.115 ; \mathrm{F}[1,18]=16.481$, $\mathrm{p}=0.001, \eta^{2}=0.022$; and $\mathrm{F}[1,18]=16.716, \mathrm{p}=0.001$, $\eta^{2}=0.036$, respectively) than the $10-\mathrm{cm}$ stairway walking training group, but not on the gastrocnemius.

\section{DISCUSSION}

In the present study, we found that the group with stair walking training at $15-\mathrm{cm}$ high had higher rectus femoris, biceps femoris, and tibialis anterior activities than the group with stair walking training at $10-\mathrm{cm}$ high. This finding possibly indicates that the step height of $15 \mathrm{~cm}$ is more effective than the step height of $10 \mathrm{~cm}$ for improving the muscle strength of paralyzed lower leg muscles.

Roh and Park ${ }^{[17]}$ analyzed body movements during stair climbing of elderly. The $18-\mathrm{cm}$ step height required an excessive force while ascending and descending the stairs, and reported that it was difficult to maintain body balance due to the weakness of the body's core muscle. They strongly advocated designing the height of stairs for elderly individuals to be $15-\mathrm{cm}$ or less. In another study, $\mathrm{Kim}^{[33]}$ reported that vertical movements of 9.7 to $12.4-\mathrm{cm}$ in the ankle joint were observed in normal adults while climbing stairs. Nadeau et al. ${ }^{[12]}$ also reported that knee joint extensor moments were up to two times greater during stair ascending than during level-ground walking. Reeves et al. ${ }^{[34]}$ showed that ankle plantar flexor and knee extensor played a pivotal role in moving the body's mass upward and forward toward the next step during stair ascending. During stair climbing, the rectus femoris and tibialis anterior showed a higher muscle activity than the medial part of the gastrocnemius, which is the posterior muscle, and the biceps femoris showed the highest activity in the one-legged stance phase during stair walking. ${ }^{[19]}$ In addition, previous studies demonstrated that, while stair ascending, a strong knee extensor force was generated to lift the body upward. ${ }^{[1]}$ Considering the results of these studies, ${ }^{[11,12,19,34]} 15-\mathrm{cm}$ stair walking training was more effective in increasing the rectus femoris muscle activity than $10-\mathrm{cm}$ stair walking training, and the $15-\mathrm{cm}$ stair height was possibly considered the height that facilitates normalization of the muscle contraction mechanism of the paralyzed lower limb and supports postural control of the one-legged 
stance phase. Therefore, the $15-\mathrm{cm}$ stair height could be considered a more convenient height for moving the body upward than a $10-\mathrm{cm}$ stair height.

Furthermore, stair walking training was effective in increasing the activity of the rectus femoris in individuals in their twenties, and it was effective in increasing the muscle activity of the biceps femoris in elderly individuals. ${ }^{[35,36]}$ These previous studies showed that the knee joint flexor and extensor activities are active while walking on the stairs, which support the results of our study that a $15-\mathrm{cm}$ stair height is more effective for lower limb muscle activation than the 10-cm stair height.

Paralyzed leg due to a stroke makes the dorsiflexion inadequate in the swing phase of gait. Therefore, it is important to induce tibialis anterior muscle contraction in rehabilitation training program for improvement of the gait function. ${ }^{[37]}$ Hemiplegia due to stroke changes the normal balance of cortical excitability of the cerebral hemisphere. Therefore, it needs to improve walking speed to improve cortical excitability, and tibialis anterior muscle activation is needed to improve walking speed. ${ }^{[38]}$ The results of the study comparing the TA muscle activity according to the gait condition for normal adults suggested that the training to increase the TA muscle activity during the stair climbing had a positive effect on making the stair walking more efficient. ${ }^{[39]}$ Therefore, these previous studies positively support the results of this study that a $15-\mathrm{cm}$ stair height is more effective for tibialis anterior muscle activation than the $10-\mathrm{cm}$ stair height. ${ }^{[37-39]}$

Nonetheless, this study has some limitations. First, it failed to identify the effects of continuous intervention after the six-week intervention. In this study, the effect sizes for the main variables (step height) were $0.12,0.02$, and 0.04 , respectively, indicating medium or small explanatory power. ${ }^{[31]}$ These results can be attributed to the small sample size and increasing the explanatory power of the main effect by obtaining more subjects is necessary. In addition, the EMG results of the lower limb muscles measured during stair climbing is a variable which includes the active contraction of lower extremity muscle in addition to the activities of a spastic muscle. Therefore, careful attention should be paid to the clinical implementation of the EMG results in the study. Thus, it is early to apply the results of our study to a general rehabilitation program for patients with hemiparetic stroke. However, this study is clinically valuable, as it describes the training effect according to the height of stairs in stair walking training.

In conclusion, our study results suggest that stair walking training at a stair height of $15-\mathrm{cm}$ is more effective for increasing the muscle activity of the paralyzed lower limb than at a stair height of $10-\mathrm{cm}$. Therefore, stair walking training at a stair height of $15-\mathrm{cm}$ may be an effective intervention to improve the paralyzed leg strength of patients with stroke.

\section{Declaration of conflicting interests}

The authors declared no conflicts of interest with respect to the authorship and/or publication of this article.

\section{Funding}

The authors received no financial support for the research and/or authorship of this article.

\section{REFERENCES}

1. Kwon MJ. The relationship between pain and quality of life in stroke patients. J Korean Soc Phys Med 2017;12:83-90.

2. Kim KD, Min KO, Shim JH, Jeong JY, Kim YE, Kim $\mathrm{KY}$, et al. Effects of motor skill learning on balance and coordination in excitoxicity induced cerebellar injury model of rat. J Korean Soc Phys Med 2010;5:455-65.

3. Kelley RE, Borazanci AP. Stroke rehabilitation. Neurol Res 2009;31:832-40.

4. Bonnyaud C, Zory R, Pradon D, Vuillerme N, Roche N. Clinical and biomechanical factors which predict timed up and down stairs test performance in hemiparetic patients. Gait Posture 2013;38:466-70.

5. Engardt M, Knutsson E, Jonsson M, Sternhag M. Dynamic muscle strength training in stroke patients: effects on knee extension torque, electromyographic activity, and motor function. Arch Phys Med Rehabil 1995;76:419-25.

6. Sharp SA, Brouwer BJ. Isokinetic strength training of the hemiparetic knee: effects on function and spasticity. Arch Phys Med Rehabil 1997;78:1231-6.

7. Celinskis D, Grabiner MD, Honeycutt CF. Bilateral early activity in the hip flexors associated with falls in stroke survivors: Preliminary evidence from laboratory-induced falls. Clin Neurophysiol 2018;129:258-64.

8. Wiley ME, Damiano DL. Lower-extremity strength profiles in spastic cerebral palsy. Dev Med Child Neurol 1998;40:100-7.

9. Meyer P, Kayser B, Mach F. Stair use for cardiovascular disease prevention. Eur J Cardiovasc Prev Rehabil 2009;16:S17-8.

10. Stacoff A, Diezi C, Luder G, Stüssi E, Kramers-de Quervain IA. Ground reaction forces on stairs: effects of stair inclination and age. Gait Posture 2005;21:24-38.

11. Riener R, Rabuffetti M, Frigo C. Stair ascent and descent at different inclinations. Gait Posture 2002;15:32-44.

12. Nadeau S, McFadyen BJ, Malouin F. Frontal and sagittal plane analyses of the stair climbing task in healthy adults aged over 40 years: what are the challenges compared to level walking? Clin Biomech (Bristol, Avon) 2003;18:950-9. 
13. Conway ZJ, Silburn PA, Blackmore T, Cole MH. Evidence of compensatory joint kinetics during stair ascent and descent in Parkinson's disease. Gait Posture 2017;52:33-9.

14. Dean CM, Richards CL, Malouin F. Task-related circuit training improves performance of locomotor tasks in chronic stroke: a randomized, controlled pilot trial. Arch Phys Med Rehabil 2000;81:409-17.

15. Seo KC, Kim HA. The effects of gait ability in the stroke patients after stair gait exercise and lamp gait exercise. J Korean Soc Phys Med 2013;8:397-406.

16. Yun HJ, Oh DW, Kim KH, Ki KI. Comparison of different ground contacts on muscle activation of post-stroke hemiparesis during setp-up activity. J Korean Propri Neuromuscul Facilit Assoc 2010;8:9-15.

17. Roh CG, Park BJ. A study on the design criteria of pedestrian facility (stairs) by motion analysis of walking parameters in the edlerly. J Korean Soc Transp 2017;35:396-408.

18. Kim YS. Muscle activation patterns of stair gait in hemiparetic patients using surface electromyography. J Adap Phys Activ 2006;14:1-15.

19. Kim YS, Kim EJ, Seo CJ. The comparative analysis of EMG and gait patterns of lower extremities during going up stairs and down. Korean J Phys Edu 2006;45:535-45.

20. Park SK, Cheon DW. Analysis of muscle activity with lower extremity during stairs and ramp ascending of hemiplegic patients. J Korean Soc Phys Ther 2012;24:247-52.

21. Ghotbi N, Nakhostin Ansari N, Naghdi S, Hasson S. Measurement of lower-limb muscle spasticity: intrarater reliability of Modified Modified Ashworth Scale. J Rehabil Res Dev 2011;48:83-8.

22. Chiu SL, Chang CC, Dennerlein JT, Xu X. Age-related differences in inter-joint coordination during stair walking transitions. Gait Posture 2015;42:152-7.

23. Ng SS, Ng HH, Chan KM, Lai JC, To AK, Yeung CW. Reliability of the 12-step ascend and descend test and its correlation with motor function in people with chronic stroke. J Rehabil Med 2013;45:123-9.

24. Chan PP, Si Tou JI, Tse MM, Ng SS. Reliability and validity of the timed up and go test with a motor task in people with chronic stroke. Arch Phys Med Rehabil 2017;98:2213-20.

25. Huang YJ, Lin GH, Lee SC, Chen YM, Huang SL, Hsieh CL. Group- and individual-level responsiveness of the 3-point berg balance scale and 3-point postural assessment scale for stroke patients. Arch Phys Med Rehabil 2018;99:529-33.

26. Prilutsky BI, Petrova LN, Raitsin LM. Comparison of mechanical energy expenditure of joint moments and muscle forces during human locomotion. J Biomech 1996;29:405-15.
27. Yang Y, Rendig S, Siegel S, Newport DF, Cherry SR. Cardiac PET imaging in mice with simultaneous cardiac and respiratory gating. Phys Med Biol 2005;50:2979-89.

28. Tang W, Zhang X, Tang X, Cao S, Gao X, Chen X. Surface electromyographic examination of poststroke neuromuscular changes in proximal and distal muscles using clustering index analysis. Front Neurol 2018;8:731.

29. Hammond CA, Hatfield GL, Gilbart MK, Garland SJ, Hunt MA. Trunk and lower limb biomechanics during stair climbing in people with and without symptomatic femoroacetabular impingement. Clin Biomech (Bristol, Avon) 2017;42:108-14.

30. Tomko PM, Colquhoun RJ, Magrini MA, Muddle TWD, Jenkins NDM. Global electromyographic signal characteristics depend on maximal isometric contraction method in the knee extensors. J Electromyogr Kinesiol 2018;42:111-6.

31. Pierce CA, Block RA, Aguinis H. Cautionary note on reporting eta-squared values from multifactor ANOVA designs. Edu Psychol Measur 2004;64:916-24.

32. Timothy RL, Craig RH. Eta squard, partial eta squared, and misreporting of effect size in communication research. Hum Commun Res 2002;28:612-25.

33. Kim JS. A study on the vertical and horizontal movements of lower extremities by an analysis of walking and stepping movements. J Appl Sci Technol 1995;4:105-17.

34. Reeves ND, Spanjaard M, Mohagheghi AA, Baltzopoulos V, Maganaris CN. Older adults employ alternative strategies to operate within their maximum capabilities when ascending stairs. J Electromyogr Kinesiol 2009;19:e57-68.

35. Kim KM, Shin JJ, Park HM, Lee DY. The effect of thigh muscle activity for stair walking and stepper strengthening exercise in university students. J Korea Academia-Industrial Cooper Soc 2014;15:936-8.

36. Min BC, Kim JH, Jeon KJ, Lee DH, Kim JS. EMG fatigue comparative study of stair ascending and descending. Korea Ind Sys Engineer 2006;2:73-6.

37. Burridge JH, Taylor PN, Hagan SA, Wood DE, Swain ID. The effects of common peroneal stimulation on the effort and speed of walking: a randomized controlled trial with chronic hemiplegic patients. Clin Rehabil 1997;11:201-10.

38. Palmer JA, Needle AR, Pohlig RT, Binder-Macleod SA. Atypical cortical drive during activation of the paretic and nonparetic tibialis anterior is related to gait deficits in chronic stroke. Clin Neurophysiol 2016;127:716-23.

39. Le M, Yuan Y, Na C, Rong S, Le L. Effect of different terrains on onset timing, duration and amplitude of tibialis anterior activation. Biomed Sign Proc Contr 2015;19:115-21. 